\title{
Significant Mutations of Hantaviruses Contribute to Its Cytotoxicity
}

\author{
Alinur Sayat \\ Haileybury Almaty, 112 al-Farabi Avenue, Almaty, 050040, Kazakhstan; 10sayat1@haileyburyalmaty.kz
}

ABSTRACT: Hantaviruses are pathogens that belong to the Bunyaviridae family which are segmented negative-strand RNA viruses. Just as many other negatively stranded RNA viruses, its RNA-dependent RNA polymerase (RdRp), also referred to as $\mathrm{L}$ protein of hantaviruses, lacks capping activity, which enhances mRNA processing, mRNA export, and translation. Hantaviruses, on the other hand, employ the use of "cap snatching", in which 5' capped oligonucleotides are cleaved from infected cell transcripts and used as primers to initiate the viral mRNA synthesis and it is known that the cytotoxicity of a hantavirus depends on the effectiveness of its cap-snatching domain. In this article, the correlation between the viral toxicity and its cap-snatching domain (first 220 amino acids of the $\mathrm{L}$ protein) is explored. As it has been found by aligning the RdRp residue cap-snatching domains, certain mutations can completely reduce the viral cytotoxicity.

KEYWORDS: Computational Biology and Bioinformatics; Computational Biomodeling; Sequence alignment; Cap-snatching domain.

\section{- Introduction}

Hantaviruses belong to the Bunyaviridae family, which is a group of segmented negative-strand RNA viruses that include ones which cause severe human diseases. ${ }^{1-3}$ Hantaviruses get significant attention as emerging pathogens which are expanding their global distribution. ${ }^{4-7}$ In Asia, the prototypic Hantaan virus is a causative agent for hemorrhagic fever with the renal syndrome with fatality rates of about $3 \%$. In South and North Americas, the hantaviruses Sin Nombre and Andes are associated with hantavirus cardiopulmonary syndrome with up to 40 $\%$ mortality. ${ }^{7-12}$ There is currently no licensed vaccine against hantaviruses and therapeutic options are limited. ${ }^{13}$

Just as many other RNA viruses, hantavirus RNA dependent RNA polymerase uses a unique "cap snatching" mechanism for transcription initiation, as viral mRNAs are around $100 \mathrm{nu}-$ cleotides shorter than parent genomic viral RNAs, and lack a poly-A tail. ${ }^{1-3}$ During the cap-snatching process, $10-14$ nucleotides' long $5^{\prime}$-capped oligonucleotides are cleaved from host cell transcripts and used as primers to initiate the viral mRNA synthesis, following a prime-and-realign mechanism. Viral mRNAs are translated into the cell cytoplasm by the host cell translation machinery. ${ }^{4-7}$ (Figure 1)

Viral RdRp also replicates the genome through the process of complementary cRNA synthesis. Complementary cRNAs are the precise complement of genomic viral RNA (vRNA) and performance templates for the synthesis of the negative-sense viral genome. Viral assembly and maturation occur on the cell surface or on the Golgi body. Virions that mature on the Golgi body are transported to the cell surface through the vesicular secretory pathways located in the cytoplasm. As a result, new virions bud off the host cells from the cytomembrane..$^{1-3}$

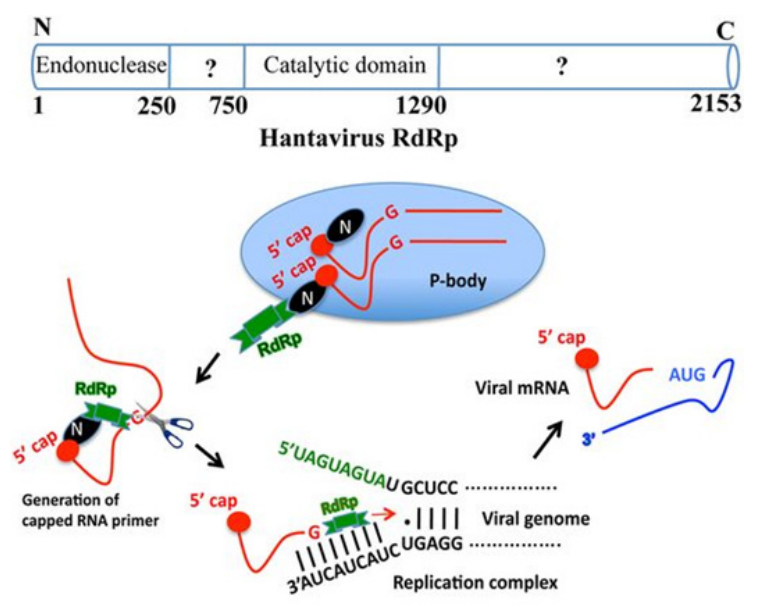

Figure 1: The cap-snatching mechanism. The diagram illustrates the cap-snatching mechanism of the RdRp and how it is involved in the viral replication in the host cell. ${ }^{14}$

\section{$R N A$-dependent $R N A$-polymerase:}

Hantavirus RdRp (RNA dependent RNA polymerase) is a large protein with a mass of $250-280 \mathrm{kDa}$. RdRp mediates both transcription and replication of the viral genome. During transcription, RdRp synthesizes viral mRNA from a negative-sense vRNA template. ${ }^{1-3}$ During replication, RdRp replicates the viral RNA genome via a cRNA intermediate. Thus, it is likely that hantavirus RdRp has multiple activities, including endonuclease (cap-snatching activity),replicase, transcriptase, and RNA helix unwinding activities. ${ }^{13}$

Due to their essential role in virus multiplication, the conserved endonucleases of RdRp of segmented negative-strand RNA virus polymerases are of great interest to basic virus research. Their enzymatic activity makes them attractive drug 
targets for therapeutic intervention. Here, previous studies were confirmed and extended, providing further evidence for the importance of the cap-snatching domain for viral toxicity. $^{13}$

\section{Importance and Hypothesis:}

In the infected host cell, cap-snatching is a mechanism of great interest as it is responsible for the initiation of virus replication. Consequently, the interruption in cap-snatching through certain mutations in the viral $R d R p$ will inhibit virus replication and will likely improve the prognosis of hantavirus disease. Our studies present the mutations of the cap-snatching domain of five different hantaviruses, as it was believed that certain mutations would completely reduce the viruses' cytotoxicity or make them even more dangerous. The study was executed to give a deeper insight into the RdRp endonuclease domain and its mutations. Identification of certain mutations will potentially reveal a novel therapeutic target for combating this viral illness.

\section{Methods \\ Sequence alignment:}

The sequence alignment was executed by web platforms such as NCBI Protein Blast (National Institute of Health, Maryland,US) and Clustal W (EMBL-EBI, Cambridgeshire, $\mathrm{UK}){ }^{26}$ The sequences of Andes and Maporal virus were compared to Hantaan virus endonuclease (access ID is 5ize at Protein Data Bank (PDB) RCSB, US). The first 220 amino acids in the sequence were taken out to the whole $\mathrm{L}$ protein sequence, to focus on the cap-snatching domain only. Only then they were aligned and checked for mutations. The reference codes used for L Protein Cap-snatching domains of hantaviruses are as follows: Puumala virus (GenBank: BAJ14125.1), Hantaan virus (GenBank: AAG10042.1), Maporal virus (NCBI Reference Sequence: YP_009362282.1), Tula virus (NCBI Reference Sequence: NP_942124.1), Andes virus (GenBank: AAG22533.4).

\section{Swiss-Model:}

The Andes hantaviruses (PDB: 5hsb) and Hantaan virus (PDB: 5ize1.A) templates for MAPV modeling were selected in SWISS-MODEL (Swiss Institute of Bioinformatics, Basel, Switzerland) template library supported Protein Data Bank (PDB) by NCBI. ${ }^{14}$ The protein sequences were copied and aligned with the corresponding template sequences. The variable regions which include insertions and deletions were rebuilt by the fragment library. The side chains were added later. Consistent with land Protocols, the geometry of the model was maintained by a physical phenomenon. The model also can be built by using PROMOD-II. The standard of the model was estimated by QMEAN. A score closer to 0 demonstrates the best quality. The scores relatively closer -1 were not used. QMEAN consists of four components: (i) the neighboring $\mathrm{C} \beta$ atoms' interaction value, (ii) all atoms interaction, (iii) the solvation potential, (iv) the torsion potential. All scores were recorded. The similarity of organic compound alignment was estimated by the 'local quality estimate' analysis executed by the server. The values smaller than 0.6 were avoided and only the upper values were accepted. Consequently, the model was tested for the standard by comparing avoided and only the upper values were accepted. Consequently, the model was tested for the standard by comparing its QMEAN mean score to other rebuilt models. The model was illustrated with its variance. Importantly, the model was accepted if it is within the range of mean giving only one unit of ordinary deviation.

The model of hantaviruses comes with manganese atoms; therefore, it is important to pick the high-quality ligand alignment. The ligand modeling was selected by using four criteria based on the template alignment of the model: (i) the biologically relevant ligands, (ii) the ligand contact with the model, (iii) the absence of ligand clashing, (iv) the conserved sequences alignment where they interact with ligands. The loss of one of the standards accustomed assumes that the model does not satisfy the analysis. At the same time, the accuracy of the tertiary structure was estimated by the GMQE score, which ranges from 0 to 1 . The upper score shows the upper reliability of the structure.

\section{Theoretical pl/Mw:}

The calculation of theoretical $\mathrm{pl} / \mathrm{Mw}$ of each $\mathrm{N}$ terminus cap-snatching domain was executed by ExPASy pl/Mw computing tool. The first 220 amino acids in the sequence of each chosen hantavirus were applied. Protein $\mathrm{pI}$ is calculated using $\mathrm{pK}$ values of amino acids, which were defined by examining polypeptide migration between $\mathrm{pH} 4.5$ to 7.3 in an immobilized $\mathrm{pH}$ gradient gel environment with $9.2 \mathrm{M}$ and $9.8 \mathrm{M}$ urea at $15{ }^{\circ} \mathrm{C}$ or $25^{\circ} \mathrm{C}$. Protein $\mathrm{Mw}$ was calculated by the addition of average isotopic masses of amino acids in the protein and the average isotopic mass of one water molecule. Molecular weight values were given in Dalton (Da).

\section{- Results and Discussion}

Previous studies have shown the existence and the functionality of an endonuclease in the $\mathrm{N}$-terminal domain of the hantavirus genus. This research sought to demonstrate certain mutations in the cap-snatching domain of the 5 hantaviruses. It has shown that there are certain amino acids, the mutations of which, will completely decrease the toxicity of the virus and consequently its lethality.

First, the $\mathrm{N}$ terminus (first 220 amino acids of the $\mathrm{L}$ protein) of five hantaviruses were aligned, showing the evolutionary distances between them (Figure 2). This shows how Tula and Puumala viruses are closely related. They are $85.91 \%$ identical (Table 1), which would explain their similar cytotoxicity with mortality rates of less than $1 \%{ }^{15-18}$ At the same time, it was observed that about $13 \%$ of the protein sequence differentiates Puumala and Tula viruses from a toxic Andes virus which has a mortality rate of between $40 \%$ and $50 \%$ in South America. ${ }^{19}$ As a result, it can be assumed that this $13 \%$ of the protein cap-snatching domain significantly changes the cytotoxicity of the virus. This shows that by looking at those mutations that are present in one virus and do not occur in the other one, which mutations actually do affect the toxicity of the hantavirus can be determined.

A detailed alignment of five hantaviruses was executed, allowing exact mutations and differences between the highly toxic and relatively safe viruses to be seen (Figure 3 ). To illu- 
minate insignificant mutations that are very unlikely to change the functionality of the cap-snatching domain, the mutations in which the amino acid was replaced by the one with a similar functional group and properties were ignored. For instance, the tenth amino acid in the sequence is $\mathrm{K}$ in Hantaan but $\mathrm{R}$ in the other 4 hantaviruses, however, they both are positively charged, thus their functionality is very similar, and it should not strongly affect the functionality of the virus.

Table 1: The genetic distance difference in the percentage value of five hantaviruses. The table was built by the comparison of the $\mathrm{L}$ protein cap-snatching domain performed by the Clustal Omega web platform (version by 23 August 2020).

\begin{tabular}{|c|c|c|c|c|c|}
\hline & Hantaan & Andes & Maporal & Puumala & Tula \\
\hline Hantaan & 100.00 & 71.82 & 70.45 & 67.73 & 68.64 \\
\hline Andes & & 100.00 & 88.18 & 76.82 & 77.27 \\
\hline Maporal & & & 100.00 & 76.82 & 78.64 \\
\hline Puumala & & & & 100.00 & 85.91 \\
\hline Tula & & & & & 100.00 \\
\hline
\end{tabular}

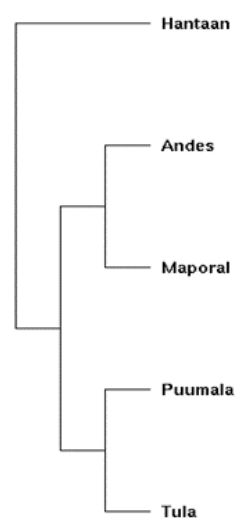

Figure 2: Phylogenetic tree of five Hantaviridae genus species and their genetic difference distance. The diagram shows the phylogenetic tree between five hantaviruses, illustrating the common ancestors based on the $\mathrm{L}$ protein alignment. The cladogram was built by the Clustal Omega web platform (version by 23 August 2020).

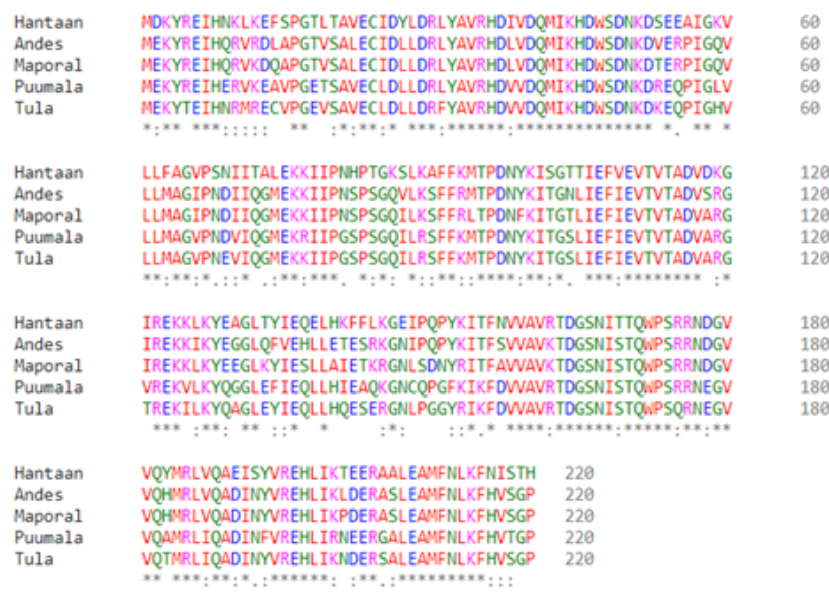

Figure 3: Alignment of the 220 amino acids of the $\mathrm{L}$ protein cap-snatching domains. The figure illustrates the alignment of the active centers of 5 Hantaviruses. The red color stands for a hydrophobic residue, green - polar and blue - negative with pink - positive. The numbers on the right side of the row tell the number of amino acids in the row, performed by the Clustal Omega web platform (version by 23 August 2020).
Next, the active sites of each of the endonuclease domains with the most conserved amino acids were defined (Figure 4). None of the mutations in the conserved amino acids were found. Therefore, it can be concluded that the observation supports previous studies which imply that one of the mutations in the conserved amino acids would fully decrease the endonuclease activity of the protein, as these amino acids ( $\mathrm{H} \mathrm{36}$, E 54, D 97, E 110, $\mathrm{T}$ 112) are bonded with manganese atoms ligands. ${ }^{20,21,13}$ This helps to better understand the nature of the mutations and their specific location in the sequence which can be linked to its significance in the efficiency of the cap-snatching domain. ${ }^{22}$

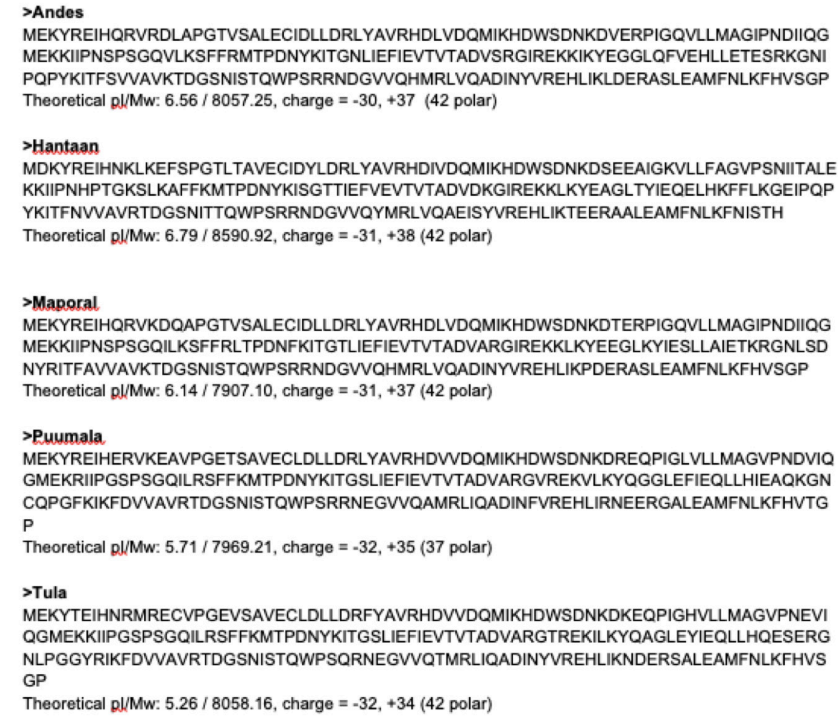

Figure 8: The protein sequence of the L Protein cap-snatching domain of five hantaviruses (220 amino acids). The diagram shows the amino sequence of the cap-snatching domains (first 220 amino acids). The connotation of each sequence above shows the isoelectric point and molecular weight of the protein sequence with the overall number of charged and polarized amino acids in the residue. This allows us to better understand the nature of the protein $\mathrm{N}$ terminus executed by the ExPASy - Compute pl/Mw tool.

Subsequently, the cap-snatching domains of both Andes and Hantaan viruses were aligned in 3D, as Andes's $\mathrm{N}$ terminus domain is evolutionarily the closest to Hantaan's domain. Moreover, both of them have a mortality of HNDV $10-12 \%$ and ANVD $30 \%$. The most functionally important mutations are shown in the diagram (Figure 5). The 3D alignment reveals that the activities of both hantaviruses are similar and considering their relatively high cytotoxic activity it can be implied that the mutations on the cap-snatching domains do indeed affect its cytotoxicity.

Andes virus is similar to the Maporal virus by $88.18 \%$, being the closest related species in the Hantaviridae genus out of the five which have been chosen for this study. However, the mortality of the Maporal virus is about $1 \%{ }^{23-26}$ All of the important mutations in the cap-snatching domain are shown Figure 6. It can be concluded that each of these mutations may decrease the toxicity of the Andes virus. 


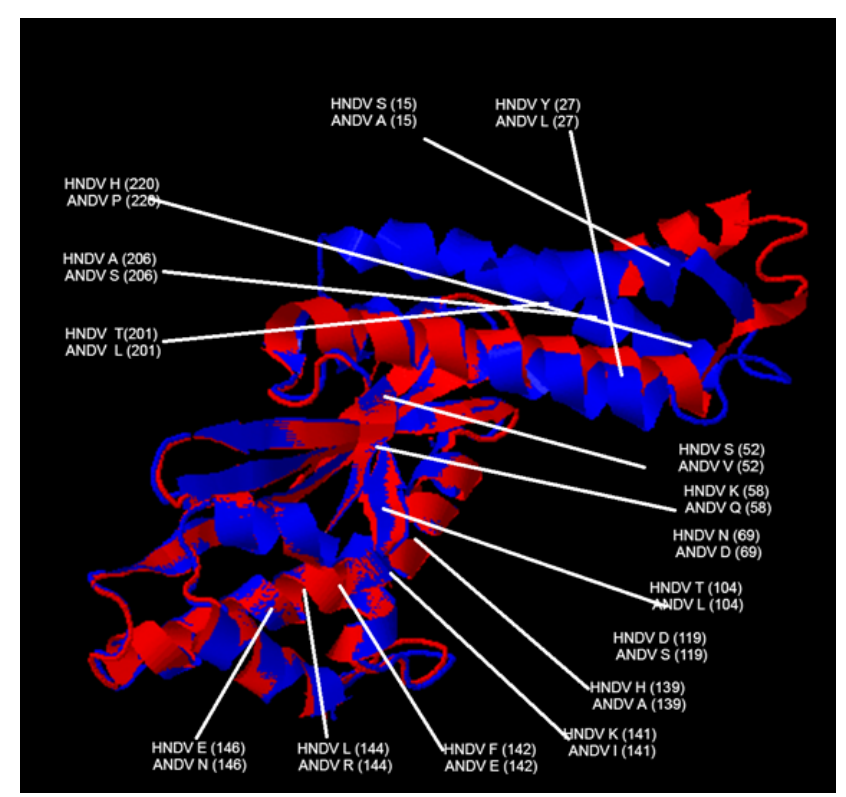

Figure 5: The 3D alignment of the Hantaan virus with Andes Virus. The diagram shows the important mutations on the aligned 3D models of the Andes virus and Hantaan virus $\mathrm{L}$ protein cap-snatching domain. The figure illustrates that the active site of the protein in both viruses is relatively similar.

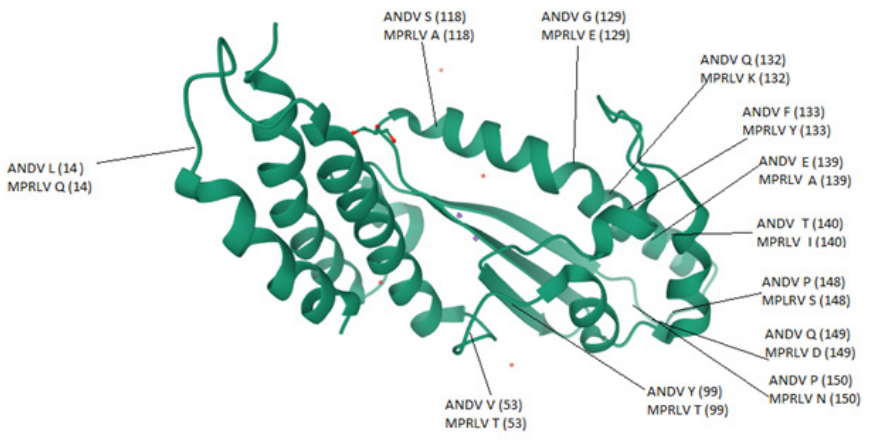

Figure 6: The comparison of the Maporal virus mutations on the Andes virus cap-snatching domain. The mutations of the Maporal hantavirus are displayed on the Andes virus cap-snatching domain. Each of these mutations significantly decreases viral toxicity.

Moreover, the Andes virus $\mathrm{N}$ Terminus model was used as a reference for Tula and Puumala viruses to demonstrate the important mutations with each of these viruses. Each of the demonstrated mutations on the Andes hantavirus cap-snatching domain reduces the cytotoxicity of the virus. This has revealed some common mutations such as ANDV E (141) and TULA H (141) with PUUM I (141).

This conclusion was revealed by executing a similar operation with Tula and Puumala virus. Tula and Andes viruses cap-snatching domains are similar with $77.27 \%$ homology. The important mutations are shown below in Figure 7.

Andes and Puumala are similar with $76.82 \%$ homology. Then their 3D models were also aligned, demonstrating the important mutations which result in such a big difference in cytotoxicity and as a result of mortality (Figure 8).

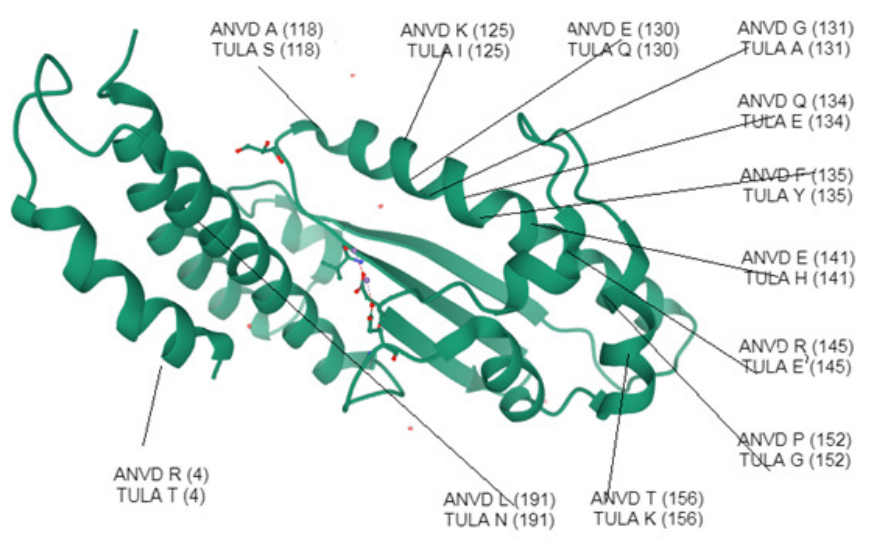

Figure 7: The comparison of the Tula virus mutations on the Andes virus 3D model. The Protein Data Bank Fasta file for the ANDV L Protein capsnatching domain was used as a reference model to build the TULA virus cap-snatching domain. The model represents the mutations by comparing the number of the amino residue and the hantavirus revealing the mutations which decrease the toxicity of the virus.

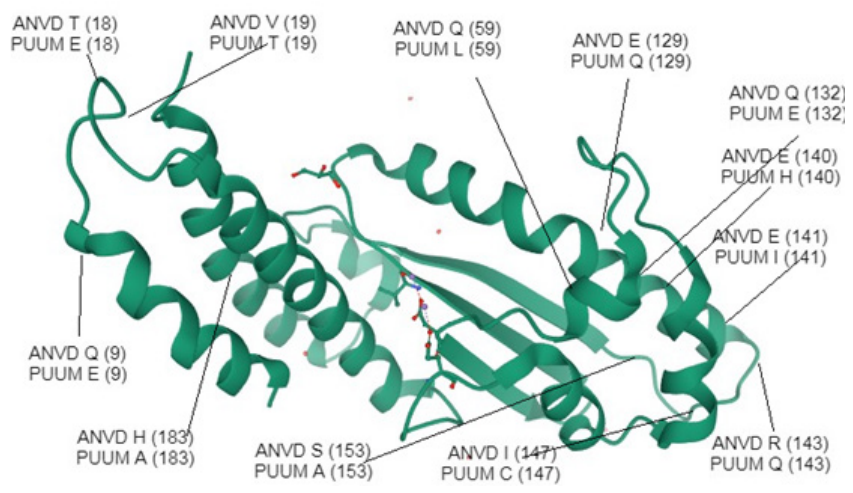

Figure 8: The comparison of the Puumala virus mutations on the Andes virus 3D model. The Protein Data Bank Fasta file for the ANDV L Protein cap-snatching domain was used as a reference model to build the PUUM virus cap-snatching domain. The model represents the mutations that appeared in the PUUM domain confirmation.

\section{- Conclusions}

Hantaviruses are viruses that belong to the Bunyaviridae family which are segmented negative-strand RNA viruses. This means that its RNA-dependent RNA polymerase (RdRp) lacks capping activity and instead it makes use of a "cap-snatching" mechanism in which 5' capped oligonucleotides are cleaved from infected cell transcripts and used as primers to initiate the viral mRNA synthesis and it is known that the cytotoxicity of a hantavirus depends on its effectiveness of cap-snatching domain. According to our hypothesis, there may be certain mutations in the cap-snatching domain of the hantaviruses which would completely reduce the cytotoxicity of the virus or make it hypertoxic. Our experiment has aligned the viruses' endonuclease domains and demonstrated the most significant mutations in the amino sequences. By comparing the toxicity of the virus, which is linked to its mortality, it could be concluded that these mutations either reduce or increase their cytotoxicity, which proves our hypothesis. 


\section{Acknowledgements}

I would like to thank my teacher Mr. Sultan Mussakhan, who has been supervising the project and sharing his experience with me. In addition, I would like to express my gratitude to my biology teachers Mrs. Carolyn Neylon, Mr. Giles Whittaker, and Mrs. Janice Williams for inspiring me to expand my knowledge further. Moreover, I want to thank my family, especially my parents for emotional and financial support. I would not be anywhere without them.

\section{References}

1. Walter C.T., Barr J.N. Recent advances in the molecular and cellular biology of bunyaviruses. J. Gen. Virol. 2011; 92:2467-2484. DOI: 10.1099/vir.0.035105-0.

2. Elliott R.M., Brennan B. Emerging phleboviruses. Curr. Opin. Virol. 2014;5:50-57. DOI: 10.1016/j.coviro.2014.01.011.

3. Elliott R.M. Orthobunyaviruses: Recent genetic and structural insights. Nat. Rev. Microbiol. 2014;12:673- 685. DOI: 10.1038/nrmicro3332.

4. Manigold T., Vial P. Human hantavirus infections: Epidemiology, clinical features, pathogenesis, and immunology. Schweiz. Med. Wochenschr.2014;144:w13937.DOI: 10.4414/smw.2014.13937.

5. Watson D.C., Sargianou M., Papa A., Chra P., Starakis I., Panos G. Epidemiology of Hantavirus infections in humans: A comprehensive, global over view. Crit. Rev. Microbiol. 2014;40:261-272. DOI: 10.3109/1040841X.2013.783555.

6. Heyman P., Ceianu C.S., Christova I., Tordo N., Beersma M., Joao Alves M., Lundkvist A., Hukic M., Papa A., Tenorio A., et al. A five-year perspective on the situation of hemorrhagic fever with renal syndrome and status of the hantavirus reservoirs in Europe, 2005-2010. Euro Surveill. 2011;16:15-22.

7. Vaheri A., Henttonen H., Voutilainen L., Mustonen J., Sironen T., Vapalahti O. Hantavirus infections in Europe and their impact on public health. Rev. Med. Virol. 2013;23:35-49. DOI: 10.1002/rmv.1722.

8. Jonsson C.B., Figueiredo L.T., Vapalahti O. A global perspective on hantavirus ecology, epidemiology, and disease. Clin. Microbiol. Rev. 2010;23:412-441. DOI: 10.1128/CMR.00062-09.

9. Kruger D.H., Schonrich G., Klempa B. Human pathogenic hantaviruses and prevention of infection. Hum. Vaccines. 2011;7:685-693. DOI: $10.4161 /$ hv.7.6.15197.

10.Macneil A., Nichol S.T., Spiropoulou C.F. Hantavirus pulmonary syndrome. Virus Res. 2011;162:138-147. DOI: 10.1016/j.virusres.2011.09.017.

11.Muranyi W., Bahr U., Zeier M., van der Woude F.J. Hantavirus infection. J. Am. Soc. Nephrol. 2005;16:3669-3679. DOI: 10.1681/ASN.2005050561.

12.Schmaljohn C.S., Nichol S.T. Bunyaviridae. In: Knipe D.M., Howley P.M., editors. Fields Virology. 5 th ed.Lippincott Williams \& Wilkins; Philadelphia, PA, USA: 2007. pp. 1741-1790.

13.Sylvia Rothenberger, Giulia Torriani, Maria U. Johansson, Stefan Kunz, and Olivier Engler. Conserved Endonuclease Function of Hantavirus L Polymerase.

Viruses. 2016 May; 8(5): 108. DOI: $10.3390 / \mathrm{v} 8050108$.
14.SWISS-MODEL (Swiss Institute of

Bioinformatics, Basel, Switzerland) template library supported Protein Data Bank (PDB) by NCBISwiss Institute of Bioinformatics, Biozentrum, University of Basel, Klingelbergstrasse 50/70, CH-4056 Basel / Switzerland.

15.Cheng E, Wang Z, Mir MA. 2014. Interaction between hantavirus nucleocapsid protein $(\mathrm{N})$ and RNA dependent RNA polymerase (RdRp) mutant reveals the requirement of $\mathrm{N}-\mathrm{RdRp}$ interaction for viral RNA synthesis. J Virol 88:8706-8712. Abstract/FREE FullText.

16.Vapalahti O, Mustonen J, Lundkvist Å, Henttonen $\mathrm{H}$, Plyusnin A, Vaheri A Hantavirus infections in Europe. Lancet Infect Dis. 2003;3:653-61 10.1016/S1473 3099(03)00774-6.

17.Schmaljohn C, Hjelle B Hantaviruses: a global disease problem. Emerg Infect Dis. 1997;3:95-104 10.3201/eid0302.970202.

18.Settergren B Clinical aspects of nephropathia epidemica (Puumala virus infection) in Europe: a review. Scand J Infect Dis. 2000;32:125-32 10.1080/003655400750045204.

19.Schönrich G, Rang A, Lütteke N, Raftery M, Charbonnel N, Ulrich R Hantavirus-induced immunity in rodent reservoirs and humans. Immunol. Rev. 2008;225:163-89 10.1111/j.1600-065X.2008.00694.x

20."Andes Virus - an overview | ScienceDirect Topics". www.sciencedirect.com. Retrieved 2019-03-07.

21.Kabsch W.S. Dictionary of protein secondary structure: Pattern recognition of hydrogen-bonded and geometrical features. Biopolymers. 1983;22:2577-2637

22.Jones D.T. Protein secondary structure prediction based on position-specific scoring matrices. J. Mol. Biol. 1999;292:195-202. DOI: 10.1006/jmbi.1999.3091.

23.Mussakhan S. (Master's Thesis). (2018) L PROTEIN N-TERMINUS BASED ASSAY DEVELOPMENT FOR ANDES VIRUS (M.Sc., Nazarbayev University).

24.Kristin K. Buys, Kie-Hoon Jung, Donald F. Smee, Yousuke Furuta,b and Brian B. Gowena,* (2011) May 12. DOI: 10.3851/IMP1729 "Maporal virus as a surrogate for pathogenic New World hantaviruses and its inhibition by favipiravir" DOI: 10.3851/IMP1729 PMCID: PMC3243073.

25.Papa A. Dobrava-Belgrade virus: Phylogeny, epidemiology, disease. Antivir. Res. 2012;95:104-117. DOI: 10.1016/j.antiviral.2012.05.011.

26.Vaheri A., Strandin T., Hepojoki J., Sironen T., Henttonen H., Makela S., Mustonen J. Uncovering the mysteries of hantavirus infections. Nat. Rev. Microbiol. 2013;11:539-550. DOI: 10.1038/nrmicro3066.

\section{- Author}

Mr Alinur Sayat is a student at Haileybury Almaty, Kazakhstan. He wants to forge his career path in the biological sciences.

$\mathrm{He}$ is an active participant in research, science fairs, and competitions. Alinur, as a student, follows the highest standards of education and always looks forward to new opportunities. 